\title{
Case Report: Hyperglycemia-an Ominous Symptom of Disease Course of Covid-19 in Full-term Newborn
}

ISSN: 2639-0531

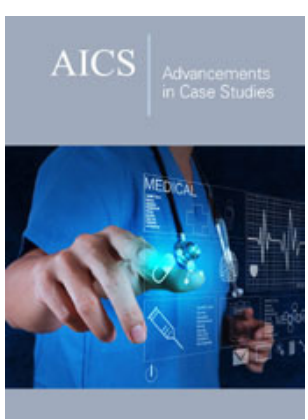

*Corresponding author: Ryszard Lauterbach, Department of Neonatology, Jagiellonian University Medical College, Poland

Submission: 漈 July 15, 2021

Published: 梅 July 26, 2021

Volume 3 - Issue 2

How to cite this article: Lauterbach $\mathrm{R}$, Pawlik D, Ulatowska Białas M, Lisowska Miszczyk I and Kacprzak M. Case Report: Hyperglycemia-an Ominous Symptom of Disease Course of Covid-19 in Fullterm Newborn. Adv Case Stud. 3(2). AICS.000556. 2021.

DOI: 10.31031/AICS.2021.03.000556

Copyright@ Lauterbach R, This article is distributed under the terms of the Creative Commons Attribution 4.0 International License, which permits unrestricted use and redistribution provided that the original author and source are credited.

\author{
Lauterbach $\mathbf{R}^{1 *}$, Pawlik $\mathbf{D}^{1}$, Ulatowska Białas $\mathbf{M}^{2}$, Lisowska Miszczyk $\mathbf{I}^{1}$ and \\ Kacprzak $\mathbf{M}^{3}$ \\ ${ }^{1}$ Department of Neonatology, Jagiellonian University Medical College, Poland \\ ${ }^{2}$ Chair and Department of Patho-morphology, Jagiellonian University Medical College, Poland, \\ ${ }^{3}$ MEDGEN Medical Centre, Poland
}

\begin{abstract}
The first four weeks after birth seem to be a relatively safe period in relation to the first pandemic of the century. Although SARS-CoV-2 can be transmitted from the mother to the newborn through droplets, the clinical symptoms are usually nonsignificant and uncomplicated. It is especially truth when SARSCoV-2 infection concerns the full-term newborn. We present the fatal disease course of COVID-19 in full term newborn delivered vaginally with appropriate weight and with Apgar score 10. On the fourteenth day of life, neonate rapidly deteriorated and within the next three days died of hyperglycemia that stimulated an inflammatory reaction.
\end{abstract}

Keywords: SARS CoV-2; COVID-19; Newborn; Hyperglycemia; Inflammatory response

\section{Introduction}

The disease course of COVID-19 is usually mild and self-limiting in term newborns, but they may also develop severe symptoms. It is particularly observed in infants with underlying comorbidities. We present a case of 14 days-old full-term newborn, male with birth weight 3680 g, delivered in $39^{\text {th }}$ week of pregnancy with Apgar score 10. Previously healthy, he was discharged from hospital on $4^{\text {th }}$ day of life with a $200 \mathrm{~g}$ weight gain. After 10 days, neonate rapidly deteriorated with poor feeding, effort dyspnea and significantly lowered muscular tonus. Five days prior to acute worsening of child condition, mother reported smell disturbances, but no presence of fever, cough, or nasal congestion.

\section{Clinical findings, diagnostic assessment, and therapeutic intervention}

Infant was transported by an ambulance to an emergency department with symptoms of dehydration, progressive respiratory insufficiency, and loss of consciousness. Moreover, the weight gain of infant between the discharge from neonatal ward and the admission to emergency department (10 days) was $100 \mathrm{~g}$. Neonatal resuscitation and then intubation and conventional mechanical ventilation (SIMV) with inspired oxygen fraction titrated up to 0.60.7 was provided. Infant temperature was $37.8{ }^{\circ} \mathrm{C}$, heart rate of 190 beats per minute and mean blood pressure of $32 \mathrm{mmHg}$. Skin perfusion was significantly disturbed with capillary refill time above 4 seconds. Initial arterial blood gas analysis showed metabolic acidosis (pH7.15, BE $(-10,4)$, lactate $11 \mathrm{mmol} / \mathrm{L})$ and an initial point-of-care blood glucose was $185 \mathrm{mg} /$ $\mathrm{dL}$. White and red blood cells as well as platelet count, showed normal values. Other main laboratory findings in the neonate like C-reactive protein, procalcitonin, liver enzymes (AST, ALT) and electrolytes concentrations also presented normal values. Point-of-care echocardiography showed an increased resistance in pulmonary arteries circulation and no congenital cardiac malformations. In the emergency department the patient received a $20 \mathrm{~mL} /$ 
$\mathrm{kg}$ normal saline bolus, and the fluid infusion was continued in a dose of $10 \mathrm{ml} / \mathrm{kg} / \mathrm{h}$. Furthermore, after cardiologist's suggestion, dopamine $(5 \mathrm{ug} / \mathrm{kg} / \mathrm{min})$ and corotrope $(0.75 \mathrm{ug} / \mathrm{kg} / \mathrm{min})$ was started. On admission to the emergency department, as per current hospital policy, infant nasopharyngeal swab was tested for the presence of SARS-CoV-2 RNA and real-time polymerase chain reaction (RT-PCR) was performed. Both the E and N2 genes were detected in nasopharyngeal swab. Child was then transferred to the neonatal intensive care unit (NICU).

On arrival to the NICU, the patient was persistently tachycardic with heart rates ranging from 190 to 210 BPM and periodically increasing to $240 \mathrm{BPM}$. He was successfully treated with adenosine and infusion of $\mathrm{MgSO}_{4}$ which improved the patient's heart rate to 170-190 BPM over the next 2 hours. Total parenteral nutrition was introduced, and mechanical ventilation (SIMV) was continued. Antibiotics (ampicillin and gentamycin) were also administered after drawing blood culture. At the opportunity of re-intubation, the bronchoalveolar lavage fluid was collected for RT-PCR and the results for $\mathrm{E}$ and $\mathrm{N} 2$ genes SARS-CoV-2 were positive. It confirmed diagnosis of COVID-19 in newborn.

From the admission to the NICU, infant was continuously unconscious and intermittently presented with irritability, hypotonia or hypertonia and opisthotonos. Routine blood tests (including glucose, C-reactive protein, procalcitonin, D-Dimer, ferritin, troponin I, NT-proBNP, and liver enzymes concentrations) were repeated and blood for culture was obtained (for enteroviruses and fungi). The laboratory findings showed significantly elevated blood glucose $(504 \mathrm{mg} / \mathrm{dL})$, troponin I $(682.3 \mathrm{ng} / \mathrm{mL}$-normal value $<47.3)$ and NT-proBNP $(52814 \mathrm{pg} / \mathrm{mL}$ - normal value $<125)$ concentrations with mildly elevated procalcitonin. At the same time, C-reactive protein, D-Dimer, ferritin, and liver enzymes levels were normal. The patient received continuous infusion of insulin with the increasing doses of $0.05 \mathrm{IU} / \mathrm{kg} / \mathrm{h}$ to $0.3 \mathrm{IU} / \mathrm{kg} / \mathrm{h}$, however the results of blood glucose measurements were still unsatisfactory and presented the levels between 300 and $400 \mathrm{mg} / \mathrm{dL}$. Blood culture was negative either for bacteria or fungi and enteroviruses. Unfortunately, the blood test with RT-PCR for SARS-CoV-2 genes was not available at hospital analytical laboratory.

Cerebral ultrasound examination revealed normal size of ventricles. However, the pictures of thalamus, frontal, and temporal lobes as well as cerebellum showed hyperechogenic lesions, that resembled injuries caused by inflammatory or/and ischemic trauma. The lumbar puncture was performed, and cerebrospinal fluid was analyzed with respect to bacteria, fungi, enteroviruses, glycorrhachia, leukocytes count and proteins concentration. A significantly increased number of leukocytes $\left(2000 / \mathrm{mm}^{3}\right)$ and raised proteins $(967 \mathrm{mg} / \mathrm{dL}$ ) and glucose concentrations $(132 \mathrm{mg} /$ dL) were found. Cerebrospinal fluid culture was negative for bacteria, fungi, and enteroviruses. However, the cerebrospinal fluid was not tested with RT-PCR for SARS-CoV-2 genes. Despite negative results of the blood and cerebrospinal cultures, an infusion of wide spectrum antibiotic (meropenem) was introduced instead of ampicillin and gentamicin. In addition to antibiotics, the infant was also treated with dopamine, corotrope, dexamethasone, pentoxifylline, insulin and diuretics.

Throughout the second day after admission to the NICU, the parameters of mechanical ventilation were reduced, and the inspired oxygen fraction lowered to 0.25 . The pulmonary ultrasounds were also normal. Subsequent echocardiography showed slight improvement in ejection fraction (EF increased from $35 \%$ to $45 \%$ ). The mean arterial blood pressure reached the value of $53 \mathrm{mmHg}$. However, diastolic disfunction of the left ventricle and a markedly increased echogenicity of the vascular wall of coronary arteries were alarming symptoms. Furthermore, the infant was deeply unconscious, and the series of convulsions appeared with the escalating frequency. Consecutive cerebral ultrasound examinations revealed a significantly increasing brain tissue oedema with extremely reduced blood perfusion, probably because of markedly elevated intracranial pressure. At the beginning of the third day after admission to the NICU, a sudden cardiac arrest occurred. Reanimation was applied promptly and after 3 minutes the cardiac function was restored. However, within the next 20 minutes the other two episodes of cardiac arrest happened and the effects of reanimation ended in therapeutic failure. As a reason of death, the brain herniation was clinically suspected.

On the ground of additional tests performed intravitally, the congenital inborn errors of metabolism were excluded. The genetic analysis of DNA microarray also did not show defects, that could explain clinical symptoms of diabetes mellitus observed in newborn. The whole exome sequencing (WES) revealed the presence of known pathogenic mutation c.271dupA (p. Cys91fs. rs549625604) in one allele of BBS10, which is known to be correlated with the Bardet-Biedl syndrome (BBS). It is an autosomal recessive multisystemic human genetic disorder characterized by six major defects including obesity, mental retardation, retinal degeneration, renal anomalies, and hypogonadism [1]. In several cases of BBS few other features such as metabolic defects, cardiovascular anomalies, hearing loss, hepatic defects and the incidence of diabetes mellitus have been reported as well. However, the heterozygous carriers of pathogenic BBS10 mutation are asymptomatic. No other potentially pathogenic variant was detected by the WES combined with the copy number variation (CNV) analysis.

\section{The results of autopsy}

At the beginning of autopsy, the presence of SARS-CoV-2 RNA in cerebrospinal fluid was tested and real-time polymerase chain reaction (RT-PCR) was performed. Both the E and N2 genes were 
detected. The results confirmed the wide-spread infection of SARS-CoV-2 in the brain tissue. Macroscopically, the brain tissue had a reduced consistency, its surface was smooth, the sulci were tightened, and the gyri were widened and flat. Histological specimens taken from various parts of the brain revealed the presence of inflammation of small meningeal and cerebral vessels (Figure 1) with endothelial cells apoptosis and multiple microthrombi (Figure 2). Small calcifications in the walls of intracerebral vessels were also observed. The entire picture of the brain corresponded to severe cerebral oedema during acute necrotizing encephalopathy.

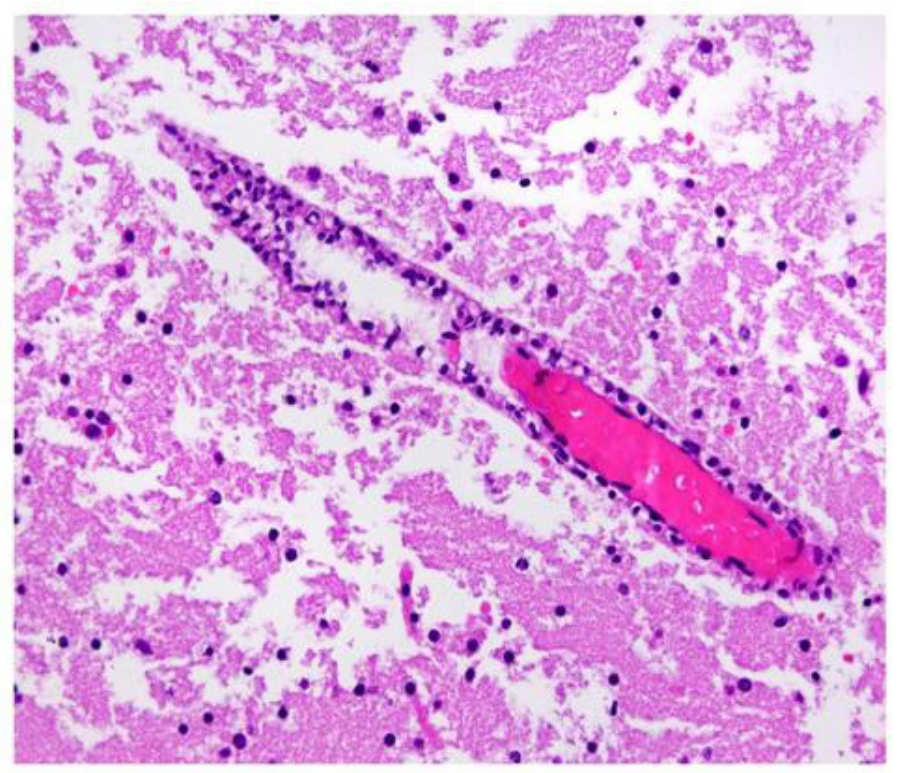

Figure 1: The presence of inflammation of small meningeal and cerebral vessels: hematoxylin-eosin, 40x.

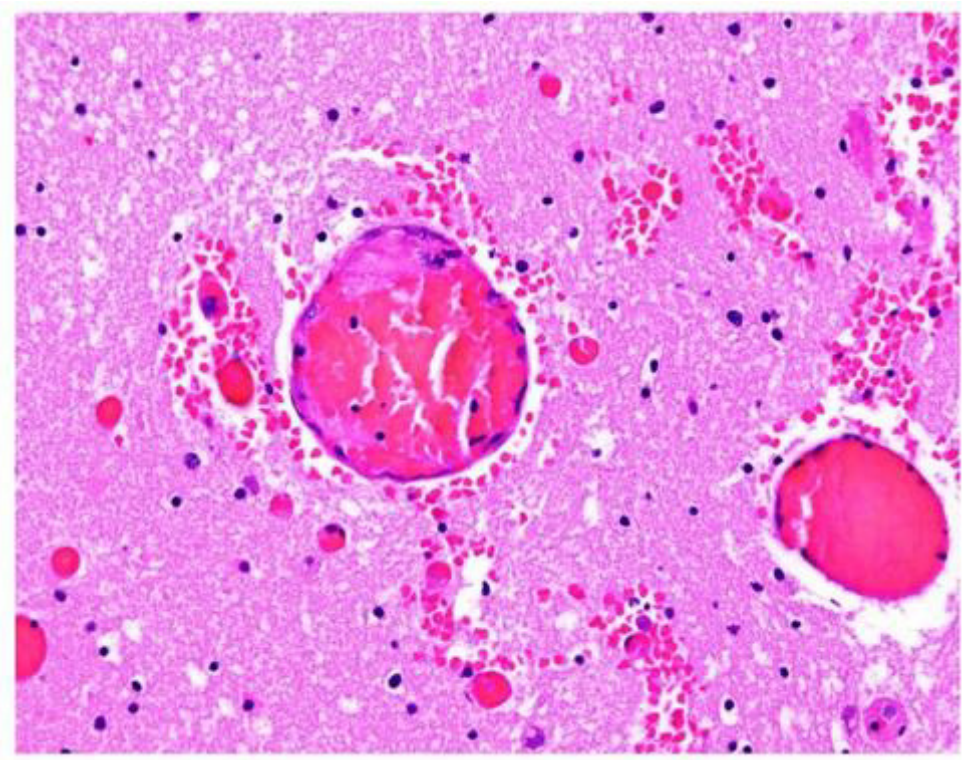

Figure 2: The endothelial cells apoptosis and multiple microthrombi: hematoxylin-eosin, 40x.

The macroscopic image of the pancreas was normal. Histological specimens stained with hematoxylin-eosin (HE) did not reveal any significant changes or abnormalities (Figure 3) but immunohistochemical reaction with neuroendocrine antibodies (both: chromogranin and synaptophysin) showed extensive damage of the pancreatic islets with significant atrophy of neuroendocrine cells in many areas of the pancreas (Figure 4). The remaining internal organs were properly developed and correctly positioned, with no visible pathologies. Severe brain damage was a direct cause of the infant's death. 


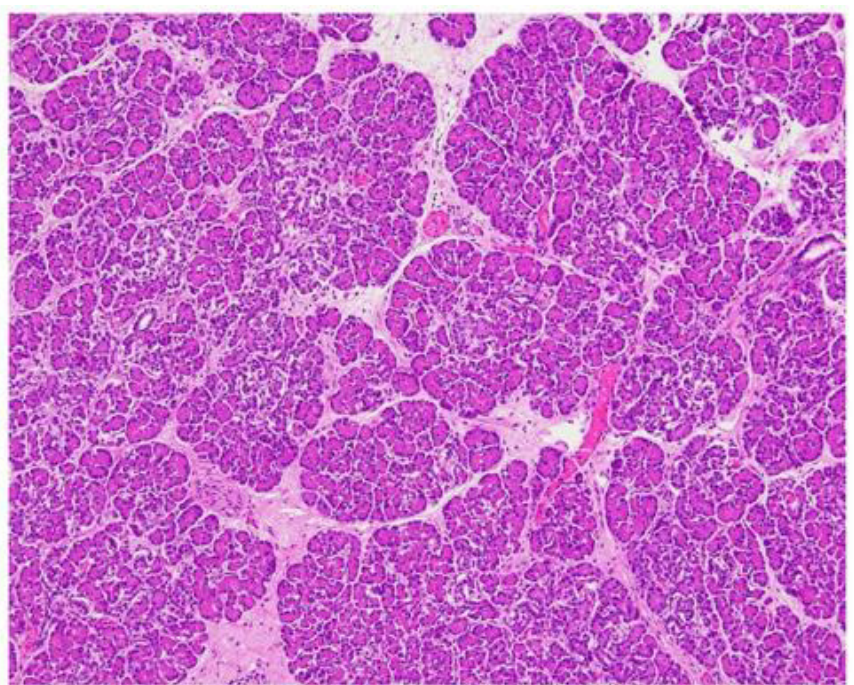

Figure 3: The histological specimens stained with hematoxylin-eosin (HE) did not reveal any significant changes abnormalities: $10 x$

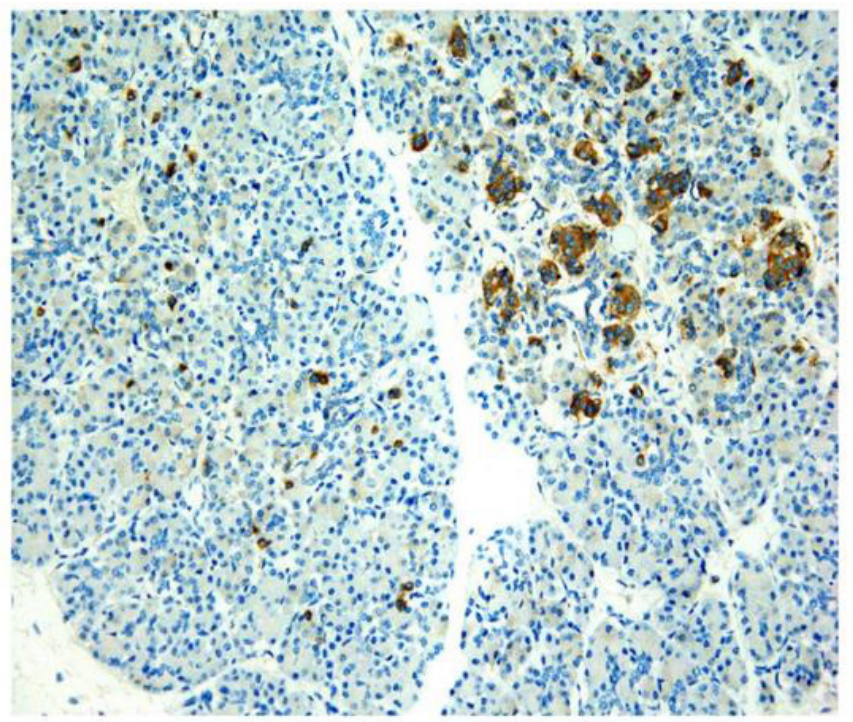

Figure 4: Immunohistochemical reaction with neuroendocrine antibodies (both: chromogranin and synaptophysin) showed extensive damage of the pancreatic islets with significant atrophy of neuroendocrine cells in many areas of the pancreas: 20x.

\section{Discussion}

The most frequently encountered presentation of COVID-19 during the neonatal period is asymptomatic or mild infection and the seemingly uncomplicated course of most cases. Our patient illustrates fatal disease course of COVID-19 in the newborn. Except for the hyperglycemia, there were no other neonatal disorders and important risk factors (e.g., bacterial, enteroviruses or fungal infections) that potentially might cause these severe clinical manifestations. Significantly increased inflammatory response, caused by SARS-CoV-2 infection, that was escalated by severe hyperglycemia, seem to be the main component of disease mechanism responsible for clinical and histological findings.

How SARS-CoV-2 affects the brain is not fully understood. A possible mechanism of entry of the virus into brain is axonal transport via the cribriform plate, adjacent to the olfactory bulb $[2,3]$. The slow circulation in the brain capillaries can also facilitate the interaction of the viral S protein with the ACE2 on brain endothelial cells. Moreover, ACE2 receptors have been also discovered in substantia nigra, ventricles, middle temporal gyrus, the posterior cingulate cortex, and the olfactory bulb [4]. 
The damaging effects of neonatal hyperglycemia on the brain during central nervous system development were reported in several studies and occurred even without concurrent bacterial or viral infections $[5,6]$. The diabetic brain exhibits neurological alterations in structure, neurotransmitters, electrophysiology, cognitive function neural density and apoptotic activity [7]. Besides the induction of pro-apoptotic proteins expression, hyperglycemia was shown to induce neuronal cell death also due to extracellular ROS generation which promoted oxidative stress and brain damage [8]. Also, it is known that hyperglycemia has a significant impact on the interactions between leukocytes and endothelial cells [9]. It was found that hyperglycemia increases the rolling, adhesion and transmigration of neutrophils and macrophages what markedly stimulates them to produce pro-inflammatory cytokines and proteolytic enzymes (elastase) [10].

The "inflammatory storm" developing during the SARSCoV-2 infection causes a significant injury of the endothelial cells and activates different processes like thrombosis, necrosis, apoptosis and efferocytosis [11]. Both, thrombosis that occurs in microcirculation and necrosis that destroys endothelial cells, devastate the blood perfusion in different organs, including brain [12]. These mechanisms cause a wide-spread injuries of central nervous system (Figure 1-2). SARS-CoV-2 was shown to bind to ACE-2 receptors through its spike proteins. ACE-2 is expressed in multiple organs, including exocrine and endocrine tissues of pancreas. Cases of pancreatitis in patients with COVID-19 have been reported in both adults and children [13]. A study of patients with diabetes strongly suggests that the localization of ACE-2 expression in endocrine part of the pancreas allows SARS coronavirus to enter and damage pancreatic islets, causing acute diabetes [14]. SARSCoV-2 have been reported to trigger transient insulin resistance and hyperglycemia.

We found the regular structure of pancreas in the tissue samples, obtained during autopsy, and stained with hematoxylineosin, however in samples assessed by immunohistochemistry, the number of pancreatic islets was significantly decreased (Figure 3,4). These microscopic images might result from SARSCoV-2 infection and pancreas damages and may explain why a severe hyperglycemia was observed in our patients from the very beginning of the disease. The extremely high blood glucose concentrations were found even despite the continuous insulin infusion, what indicated the resistance to insulin and suggested the extensive damage of pancreas.

Initially, we suspected that the reason for hyperglycemia was the congenital diabetes mellitus in newborn and as an underlying comorbidity, it severely complicated clinical course of COVID-19 in infant. However, the results of the genetic analysis did not show defects, that could explain clinical symptoms of congenital diabetes mellitus in our patient. Among the wide spectrum of symptoms of Bardet-Biedl syndrome is diabetes mellitus, however the symptoms of disease do not appear in heterozygous carriers of pathogenic BBS10 mutation. Finally, we concluded that SARSCoV-2 infection, most likely transmitted from the mother to the newborn, was the primary cause of pancreas damage, and the main reason for persistent hyperglycemia. On the ground of several mechanisms that we described above, the extremely high blood glucose concentrations significantly potentiated and escalated the inflammatory response, particularly in vascular bed of the brain and caused the critical oedema, leading to the brain herniation and death.

In summary, our intent with this case report was to caution neonatologists and pediatricians against hyperglycemia, caused by a different reason, that may stimulate the inflammatory reactions and aggravate the results of treatment of COVID-19 in full-term newborns.

\section{Ethics statement}

The patient's parents provided informed consent for the publication of this case report.

\section{Author contributions}

All authors have made a substantial and intellectual contribution to the work and approved it for publication.s

\section{References}

1. Stoetzel C, Laurier V, Davis E, Muller E, Rix J, et al. (2006) BBS10 encodes a vertebrate-specific chaperonin-like protein and is a major BBS locus. Nature Genet 38(5): 521-524.

2. Politi LS, Salsano E, Grimaldi M (2020) Magnetic resonance imaging alteration of thew brain in a patient with coronavirus disease 2019 (COVID-19) and anosmia. JAMA Neurol 77(8): 1028-1029.

3. Le Guennec L, Devianne J, Jalin L, Cao AS, Galanaud D, et al. (2020) Orbitofrontal involvement in a neuroCOVID-19 patient. Epilepsia 61(8): e90-e94.

4. Dahm T, Rudolph H, Schwerk C, Schroten H, Tenenbaum T (2016) Neuroinvasion and inflammation in viral central nervous system infections. Mediat Inflamm 2016: 8562805.

5. Rosa AP, Mescka CP, Catarino FM, Luz de Castro A, Teixeira RB, et al. (2018) Neonatal hyperglycemia induces cell death in the rat brain. Metabol Brain Dis 33: 333-342.

6. Yang CM, Lin CC, Hsieh HL (2017) High glucose-derived oxidative stressdependent Heme Oxygenase-1 expression from astrocytes contributes to the neuronal apoptosis. Mol Neurobiol 54(1): 470-483.

7. Ho FM, Liu SH, Liau CS, Huang PJ, Lin-Shiau SY (2000) High glucoseinduced apoptosis in human endothelial cells is mediated by sequential activation of c-Jun HN(2)-terminal kinase and caspase-3. Circulation 101(22): 2618-2624.

8. Rosa AP, Jacques CE, De Souza LO, Bitencourt F, Mazzola PN, et al. (2015) Neonatal hyperglycemia induces oxidative stress in the rat brain: the role of pentose phosphate pathway enzymes and NADPH oxidase. Moll Cell Biochem 403(1-2): 159-167. 
9. Booth G, Stalker TJ, Lefer AM, Scalia R (2001) Elevated ambient glucose induces acute inflammatory events in the microvasculature: effects of insulin. Am J Physiol Endocrinol Metab 280(6): E848-E856.

10. De Vries AS, Verbeuren TJ, Van De Voorde J, Lameire NH, Vanhoutte PM (2000) Endothelial dysfunction in diabetes, Br J Pharmacol 130(5): 963974.

11. McGonagle D, O Donnell JS, Sharif K, Emery P, Bridgewood C (2020) Immune mechanisms of pulmonary intravascular coagulopathy in COVID-19 pneumonia. Lancet Rheumatol 2(7): e437-445.
12. Schulert GS, Grom AA (2015) Pathogenesis of macrophage activation syndrome and potential for cytokine-directed therapies. Ann Rev Med 66: 145-159.

13. Inamdar S, Benias PC, Liu Y, Sejpal DV, Satapathy SK, et al. (2020) Prevalence, risk factors, and outcomes of hospitalized patients with COVID-19 presenting as acute pancreatitis. Gastroenterology 159(6): 2226-2228.

14. Stevens JP, Brownell JN, Freeman AJ, Bashaw H (2020) Covid-19Associated multisystem inflammatory syndrome in children presenting as acute pancreatitis. J Pediatr gastroenterol Nutr 71(5): 669-671.

For possible submissions Click below: 